\title{
Modelo numérico do transporte de nitrogênio no solo. Parte I: Desenvolvimento e teste do modelo
}

\author{
Felizardo A. Rocha ${ }^{1}$, Mauro A. Martinez ${ }^{2}$, Antônio T. Matos ${ }^{2}$, Reinaldo B. Cantarutti ${ }^{3}$ \& Joseane O. da Silva ${ }^{4}$
}

\begin{abstract}
RESUMO
Este trabalho visa realizar modificações no modelo de transporte de soluto no solo SIMASS-C, permitindo-lhe simular as concentrações do nitrato e amônio no solo, considerando-se as transformações biológicas e o efeito da temperatura e do teor de água do solo. A primeira etapa da pesquisa constou da formulação matemática e da elaboração da rotina computacional do modelo, em linguagem Delphi 7.0; na segunda etapa foram parametrizados os modelos de mineralização e nitrificação e testado o modelo SIMASS-C modificado, com base em coeficientes estatísticos pela comparação entre os dados observados e os simulados. O modelo exponencial apresentou bom ajuste aos dados de mineralização e nitrificação. A inclusão do processo de mineralização e nitrificação no modelo SIMASS-C resultou em uma melhoria na estimativa da concentração de nitrato e amônio no solo, enquanto o modelo de temperatura permitiu estimar a temperatura na camada de solo analisada e ao longo do tempo, com boa precisão.
\end{abstract}

Palavras-chave: movimento, nitrato, amônio, modelagem

\section{Numerical model of nitrogen transport in the soil. Part I: Model development and test}

\begin{abstract}
This study aims at modifying the soil solute transport model SIMASS-C, allowing it to simulate the nitrate and ammonium concentrations in the soil, considering the biological transformations and the effect of temperature and water content of the soil. The first stage consisted of mathematical formulation and elaboration of a computational routine model, in the Delphi 7.0 language. In the second stage, the parameters of the mineralization and nitrification models were fitted and the SIMASS-C model tested, considering statistical coefficients and comparison between observed and simulated data. The exponential model gave a good fitting to the mineralization and nitrification data. The incorporation of the mineralization and nitrification processes in the SIMASS-C model resulted in an improvement in the estimate of the nitrate and ammonium concentrations in the soil. The temperature model allowed to estimate temperature in soil profile with good precision.
\end{abstract}

Key words: movement, nitrate, ammonium, modeling

1 DEBI/UESB, CEP 45700-000, Itapetinga, BA. Fone: (77) 3261-8609. E-mail: felizardo@uesb.br

2 DEA/UFV, CEP 36570-000, Viçosa, MG. Fone: (31) 3899-1910. E-mail: mmauro@ufv.br

3 DPS/UFV, Fone: (31) 3899-1860. E-mail: cantarutti@ufv.br

${ }^{4}$ CCTA/UENF, CEP 28013-600, Campos dos Goytacazes, RJ. Fone: (22) 2734-4823. E-mail: joseane@uenf.br 


\section{INTRODUÇÃO}

A dinâmica do nitrogênio (N) no solo envolve processos de natureza física, química e biológica, que determinarão se o N permanecerá adsorvido na camada agricultável do solo, absorvido pelas plantas ou se moverá no solo, até atingir camadas mais profundas. O movimento e o destino desses produtos no ambiente são influenciados por processos de retenção (adsorção e absorção), de transporte (lixiviação, volatilização, escoamento superficial), de transformação biológica e pela interação desses processos (Spadotto, 2003).

Países como os Estados Unidos, Canadá, Alemanha e outros, têm desenvolvido vários modelos visando estudar a contaminação do solo e da água subterrânea; alguns merecem destaque: PRZM (Carsel et al., 1984) e LEACHM (Wagenet \& Rao, 1990); este último considera os processos de transformação biológica sofrida pelo nitrogênio no solo.

Os avanços computacionais aliados aos métodos numéricos, têm permitido o desenvolvimento de modelos que descrevem o transporte de solutos no solo, cuja vantagem principal do uso de modelos é a economia de tempo e capital investido, haja vista que possibilitam simular múltiplos cenários, ao invés de se utilizar longos períodos de observação e de coleta de dados (Azevedo et. al, 1996).

Recentemente, pesquisadores brasileiros começaram a atuar nesse campo de pesquisa; ressalta-se que os trabalhos desenvolvidos por Costa (1998) e, posteriormente, por Corrêa (2001), permitiram desenvolver um modelo computacional para simular o transporte de água e solutos no solo, considerando-se a extração de água e solutos pela cultura (SIMASS-C), sob condições de escoamento não-permanente, na vertical, mas até o momento este modelo não considera os processos de transformação biológica do nitrogênio, como a mineralização e nitrificação do amônio no solo.

A complexidade do ciclo do nitrogênio no solo torna difícil sua modelagem em termos matemáticos, haja vista que os processos observados no seu ciclo são muito dinâmicos, sendo influenciados por fatores que afetam a atividade dos microrganismos, tais como temperatura e umidade do solo (Sierra, 1997; 2002; Strong \& Fillery, 2002).

O nitrogênio das frações mais lábeis é liberado nos períodos iniciais do processo de mineralização e a estimativa da taxa de sua mineralização pode ser usada para ajustar as recomendações de adubação nitrogenada (Mengel, 1996). Do ponto de vista prático, tem-se observado que o potencial e a respectiva taxa de mineralização podem ser utilizados na predição da disponibilidade de nitrogênio às plantas, em determinado período de tempo.

Alguns autores empregam modelos empíricos e outros modelos mecanísticos para descrever essas reações biológicas sofridas pelo nitrogênio. Soluções analíticas e numéricas da equação de transporte de solutos, para várias condições iniciais e de contorno, são relatadas na literatura envolvendo uma ou mais reações do nitrogênio no solo por meio termo fonte e/ou sumidouro da equação de transporte de solutos (Mc Laren, 1969; Misra et al., 1974; Hansen et al., 1990).

Mc Laren (1973), apud Starr (1983), utilizando a equação de transporte de solutos expressou reações do nitrogênio no solo por meio de um modelo mecanístico, em que a taxa com que um substrato $\left(\mathrm{N}-\mathrm{NH}_{4}{ }^{+}\right.$ou $\left.\mathrm{N}-\mathrm{NO}_{3}{ }^{-}\right)$é utilizado pelos microrganismos depende de coeficientes que representam a taxa de crescimento, de mortalidade e de consumo de substrato pelos microrganismos. Esses coeficientes refletem os processos de mineralização e nitrificação do nitrogênio no solo.

Starr et al. (1983) empregaram a técnica de fluidos miscíveis para estudar o transporte de $\mathrm{N}_{-} \mathrm{NH}_{4}{ }^{+}$e $\mathrm{N}-\mathrm{NO}_{3}{ }^{-}$em colunas de solo. Os autores consideraram que as transformações biológicas ocorreram segundo a cinética de primeira ordem (modelo exponencial simples).

Objetivou-se, com o exposto neste trabalho: a) selecionar e parametrizar modelos que simulem o transporte de nitrato e amônio, considerando-se os efeitos da temperatura e do teor de água no solo e incorporá-los ao modelo SIMASS-C; b) acrescentar ao modelo SIMASS-C um submodelo que simule a temperatura do solo, e c) implementar modificações no modelo citado, permitindo simulações em condições de solos estratificados.

\section{MATERIAL E MÉTODOS}

O modelo computacional SIMASS-C (Simulação do Movimento de Água e Solutos no Solo, considerando a presença de Cultura), desenvolvido por Costa (1998) e aprimorado por Corrêa (2001) consiste na solução de duas equações diferenciais parciais de segunda ordem, ou seja, equação do movimento de água no solo e equação do transporte de solutos. As soluções dessas equações permitem estimar, respectivamente, os valores de potencial matricial de água e de concentração de soluto no perfil do solo. As equações são resolvidas numericamente, pelo método de diferenças finitas.

A equação diferencial que descreve o movimento de água no solo, em regime não-permanente, conhecida como equação de Richards, é:

$$
\frac{\partial \theta}{\partial t}=\frac{\partial}{\partial z}\left[K(h)\left(\frac{\partial h}{\partial z}+1\right)\right]
$$

em que:

$\theta$ - teor de água no solo, $\mathrm{cm}^{3} \mathrm{~cm}^{-3}$

K(h) - condutividade hidráulica em função do potencial de água do solo, $\mathrm{cm} \mathrm{h}^{-1}$

$\mathrm{h}$ - potencial matricial de água no solo, cm

Considerando a existência de equilíbrio linear de sorção e se acrescentando o termo fonte ou sumidouro de soluto $(\Gamma)$, obtém-se a equação de transporte de solutos descrita abaixo (Costa, 1998).

$$
\mathrm{R} \frac{\partial \mathrm{C}}{\partial \mathrm{t}}=\frac{\partial}{\partial \mathrm{z}}\left(\theta \mathrm{D} \frac{\partial \mathrm{C}}{\partial \mathrm{z}}-\mathrm{qC}\right)+\Gamma
$$

em que:

$$
\begin{aligned}
& \mathrm{R}=1+\frac{\rho_{\mathrm{s}} \mathrm{k}_{\mathrm{d}}}{\theta}=\text { fator de retardamento } \\
& \Gamma-\text { fonte ou sumidouro de soluto, } \mathrm{g} \mathrm{cm}^{-3} \mathrm{~h}^{-1}
\end{aligned}
$$


D - coeficiente de dispersão hidrodinâmica, $\mathrm{cm}^{2} \mathrm{~h}^{-1}$

C - concentração do soluto na fase líquida do solo, $\mathrm{g} \mathrm{cm}^{-3}$

$\theta$ - teor de água no solo, $\mathrm{cm}^{3} \mathrm{~cm}^{-3}$

$\mathrm{q}$ - fluxo de água no solo, $\mathrm{cm} \mathrm{h}^{-1}$

$\mathrm{K}_{\mathrm{d}}$ - coeficiente de partição do soluto, $\mathrm{cm}^{3} \mathrm{~g}^{-1}$

As modificações propostas no modelo SIMASS-C foram inseridas na equação de transporte de solutos, por meio do termo fonte ou sumidouro $(\Gamma)$, adotando-se uma equação cinética de primeira ordem para descrever as reações do nitrogênio no solo:

$$
\frac{\partial \mathrm{C}}{\partial \mathrm{t}}=\mathrm{kC}
$$

a qual se torna, após integração:

$$
\mathrm{C}=\mathrm{C}_{\mathrm{o}} \mathrm{e}^{\mathrm{k} \cdot \mathrm{t}}
$$

em que:

$\mathrm{k}$ - representa a taxa de conversão do substrato $\left(\mathrm{N}-\mathrm{NH}_{4}{ }^{+}\right.$ou N-NO$\left.{ }_{3}^{-}\right), \mathrm{g} \mathrm{cm}^{-3}$

$\mathrm{Co}$ - concentração inicial do substrato $\left(\mathrm{N}^{-\mathrm{NH}_{4}}{ }^{+}\right.$ou $\left.\mathrm{N}-\mathrm{NO}_{3}{ }^{-}\right), \mathrm{g} \mathrm{cm}^{-3}$

$\mathrm{t}$ - tempo, $\mathrm{h}$

O efeito da temperatura do solo nas transformações biológicas do nitrogênio foi descrito por um modelo analítico, proposto por van Wijk \& De Vries (1963), apud Wu \& Nofziger (1999):

$$
T(z, t)=T_{a}+\left(A_{0} e^{-z / d}\right) \operatorname{sen}\left(w t-\frac{z}{d}\right)
$$

em que:

$\mathrm{T}(\mathrm{z}, \mathrm{t})$ - temperatura média diária do solo, no tempo t e na profundidade $\mathrm{z},{ }^{\circ} \mathrm{C}$

$\mathrm{Ta}$ - temperatura média na superfície do solo, ${ }^{\circ} \mathrm{C}$

$\mathrm{A}_{0}$ - amplitude média diária da temperatura na superfície do solo, ${ }^{\circ} \mathrm{C}$

$\mathrm{w}$ - freqüência angular, $\mathrm{rad}^{-1}$

d - difusividade térmica do solo, cm

A difusividade é expressa por:

$$
d=\sqrt{\frac{2 \kappa}{C_{h} \times w}}
$$

em que:

$\kappa$ - condutividade térmica do solo, cal $\mathrm{cm}^{-1} \mathrm{~h}^{-1}{ }^{\circ} \mathrm{C}^{-1}$

$\mathrm{C}_{\mathrm{h}}$ - calor específico do solo, cal $\mathrm{cm}^{-3}{ }^{\circ} \mathrm{C}^{-1}$

$\mathrm{w}$ - freqüência angular, $\mathrm{rad}^{-1}$

A difusividade térmica depende da condutividade térmica do solo e do calor específico do solo (Elias, 2004) que, por sua vez, dependem da porosidade, do teor de água e da argila do solo (Hillel, 1980). Os valores de $C_{h}$ e $\kappa$ foram determinados conforme Wu \& Nofziger (1999).

O teor de água estimado com a solução da Eq. 1 funciona como parâmetro de entrada no modelo de temperatura (Eq. 6), que exibe, como saída, a temperatura do solo para um tempo j e profundidade i. Este modelo pressupõe que o teor de água seja constante na camada de solo analisado; portanto, para a sua aplicação sob condições de umidade variáveis no solo, considerou-se um teor médio de água no solo.

A escolha do parâmetro das funções que simulam o nitrato é função do teor de água (obtido a partir da equação de Richards) e da temperatura do solo, determinada pela Eq. 6 . Quando o íon simulado é o amônio, a taxa k assume valor negativo (sumidouro), e o modelo retira um incremento de nitrato $\left(\Delta \mathrm{NO}_{3}{ }^{-}\right)$da concentração entre os tempos tempo j e $\mathrm{j}+1$. Ao simular nitrato, o segundo termo da Eq. 8 se torna zero e vice-versa; portanto, nas simulações, o termo $\Gamma$ é expresso por:

$$
\Gamma=-\mathrm{k}_{1} \mathrm{C}+\mathrm{k}_{2} \mathrm{C}
$$

em que:

$\mathrm{k}_{1}=$ taxa de redução da concentração de $\mathrm{N}_{-} \mathrm{NH}_{4}{ }^{+}$, $\mathrm{g} \mathrm{cm}^{-3} \mathrm{~h}^{-1}$

$\mathrm{k}_{2}=$ taxa de aumento na concentração de $\mathrm{N}_{-} \mathrm{NO}_{3}^{-}$, $\mathrm{g} \mathrm{cm}^{-3} \mathrm{~h}^{-1}$

Ao simular nitrato, o segundo termo desta equação se torna zero e, simulando o amônio, o primeiro termo se torna zero.

Para implementar as modificações propostas montou-se um experimento em câmaras de incubação na qual amostras de um solo arenoso foram incubadas ao longo do tempo, variando temperatura e umidade do solo, visando parametrizar os modelos exponenciais (cinéticas de primeira ordem); posteriormente, essas cinéticas foram incorporadas à equação de transporte de solutos, termo fonte ou sumidouro, usado para simular o transporte de nitrato e amônio no perfil do solo.

Ao simular a concentração de nitrato durante o movimento de água no solo, a taxa k assume valor positivo (fonte), adicionando o incremento de concentração de nitrato $\left(\Delta \mathrm{NO}_{3}{ }^{-}\right)$ acumulado entre o tempo j e j + 1, em cada nó i (Figura 1 ).

Um segundo experimento foi montado em colunas de solo para testar o modelo SIMASS-C modificado, que possibilita simular o transporte de nitrato e amônio em profundidade e

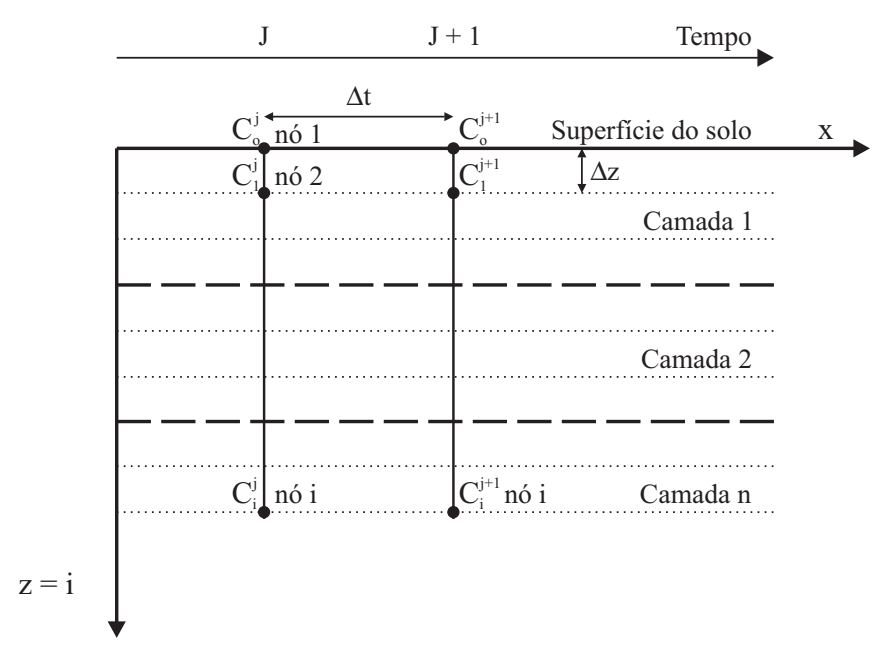

Figura 1. Discretização utilizada para resolução da equação de transporte de solutos 
ao longo do tempo, adotando-se as equações cinéticas de primeira ordem; posteriormente, compararam-se as concentrações de nitrato e amônio simuladas com os valores observados, com e sem as alterações propostas; e também comparados os resultados de temperatura do solo obtidos experimentalmente com os simulados pelo modelo proposto.

Colunas de PVC com diâmetro de 75 mm e comprimento de $40 \mathrm{~cm}$ de comprimento (solo franco-argiloso) e $60 \mathrm{~cm}$ de comprimento (solo franco-arenoso) foram construídas e preenchidas adequadamente com solo em camadas de $5 \mathrm{~cm}$, de forma a representarem densidades existente em campo; posteriormente, aplicou-se uma solução de $10 \mathrm{~mL}$ de solução de sulfato de amônio, na dose equivalente a $400 \mathrm{~kg} \mathrm{ha}^{-1}$; após 7 dias as colunas foram desmontadas e analisadas as concentrações $\mathrm{N}-\mathrm{NH}_{4}{ }^{+}$e $\mathrm{N}-\mathrm{NO}_{3}{ }^{-}$; na simulação se considerou fluxo zero na base e evaporação na superfície.

Utilizaram-se os softwares SWRC (Soil Water Retention Curve) v. 3.0, desenvolvidos por Dourado Neto et al. (2000) para ajuste do modelo de van Genhutchen (1980) e o programa computacional DISP v. 1.1., desenvolvido por Borges Júnior \& Ferreira (2006) para estimar os parâmetros de transporte para nitrato e amônio no solo.

O fator de retardamento utilizado na simulação empregando-se solo franco-argiloso, foi de 3,299 e 1,252, para o amônio e nitrato, respectivamente; enquanto para o solo arenoso foi de 2,964 e 1,223, respectivamente.

A temperatura nas colunas foi monitorada ao longo do dia, nas profundidades de $0,5,10,20,30$ e $40 \mathrm{~cm}$, para o solo franco-argiloso, e a $0,5,10,20,30,40,50$ e $60 \mathrm{~cm}$, para o solo franco-arenoso (Wierenga \& Wit, 1970); para isto foram inseridos lateralmente na coluna, sensores de temperatura do tipo LM35, modelo DZ, calibrado de fábrica, que permite leituras entre 5 e $55^{\circ} \mathrm{C}$. As características do solo utilizado na simulação estão apresentadas na Tabela 1.

Tabela 1. Características físico-hídricas do solo usado no experimento

\begin{tabular}{lcc}
\hline Características do solo & $\begin{array}{c}\text { Camada } \\
(\mathbf{0}-40 \mathrm{~cm})\end{array}$ & $\begin{array}{c}\text { Camada } \\
(\mathbf{4 0 - 6 0} \mathbf{~ c m})\end{array}$ \\
Umidade do solo na capacidade de campo $\left(\mathrm{kg} \mathrm{kg}^{-1}\right)$ & 0,211 & 0,181 \\
Umidade do solo no ponto de murcha $\left(\mathrm{kg} \mathrm{kg}^{-1}\right)$ & 0,098 & 0,115 \\
Massa espećíica $\left(\mathrm{g} \mathrm{cm}^{-3}\right)$ & 1,30 & 1,28 \\
Densidade de partículas $\left(\mathrm{g} \mathrm{cm}^{-3}\right)$ & 2,53 & 2,45 \\
\hline
\end{tabular}

Os resultados simulados foram comparados com os observados experimentalmente, avaliando-se o desempenho do modelo por meio dos coeficientes estatísticos: raiz quadrada do erro médio (RQMR), coeficiente residual de massa (CRM) e eficiência do modelo (EF), conforme Loague \& Green (1991); essas expressões são descritas nas Equações abaixo, respectivamente:

$$
\begin{aligned}
& \text { RQMR }=\left[\sum_{i=1}^{n}\left(y_{i}-\hat{y}_{i}\right)^{2} / n_{o}\right]^{1 / 2} \\
& C R M=\left[\left(\sum_{i=1}^{n} \hat{y}_{i}-\sum_{i=1}^{n} y_{i}\right) / \sum_{i=1}^{n} y_{i}\right] \\
& E F=\left[\left(\sum_{i=1}^{n}\left(y_{i}-\bar{y}\right)^{2}-\sum_{i=1}^{n}\left(\hat{y}_{i}-y_{i}\right)^{2}\right) / \sum_{i=1}^{n}\left(y_{i}-\bar{y}\right)^{2}\right]
\end{aligned}
$$

em que:

$$
\begin{aligned}
& \mathrm{y}_{\mathrm{i}} \text { - são os valores observados experimentalmente; } \\
& \hat{y}_{\mathrm{i}} \text { - são os valores simulados (preditos); } \\
& \overline{\mathrm{y}} \text { - é a média dos valores observados e no é o nú- } \\
& \quad \text { mero de observações. }
\end{aligned}
$$

\section{RESULTADOS E DISCUSSÃO}

As alterações incorporadas ao SIMASS-C, que permitiram simular o transporte de água em solos estratificados, foram realizadas na rotina computacional do programa. Com esta modificação, a entrada dos parâmetros físico-hídricos necessários à simulação passa a ser feita por nó e não mais por camadas. O programa apresenta uma opção de entrar com os parâmetros do solo e selecionar a opção de selecionar "fonte” ou "sumidouro" de soluto em cada nó existente no perfil do solo.

Como resultado o modelo exibe, em tabelas ou gráficos, o potencial matricial, a concentração de soluto (considerado acréscimo ou redução do substrato) e a temperatura do solo, ao longo do tempo.

\begin{tabular}{|c|c|c|c|c|c|c|}
\hline Temperatura $\left({ }^{\circ} \mathrm{C}\right)$ & $\begin{array}{l}\text { Teor de água } \\
\text { (U) }\left(\mathrm{kg} \mathrm{kg}^{-1}\right)\end{array}$ & $\hat{\mathbf{C}}$ & $\hat{\mathbf{k}}$ & $\mathbf{R}^{2}$ & RQMR* & $\mathrm{CRM}^{*}$ \\
\hline $5<T<20$ & $10<U>16$ & $318,991^{* *}$ & $-0,013^{\star *}$ & 85,28 & 12,95 & $-2,9 \mathrm{E}-4$ \\
\hline $5<T<20$ & $16 \leq \mathrm{U} \leq 28,5$ & $318,629 * *$ & $-0,014^{\star *}$ & 74,53 & 15,25 & $-3,8 \mathrm{E}-4$ \\
\hline $5<T<20$ & $28,5>U<37,4$ & $304,390 * *$ & 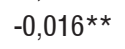 & 85,95 & 16,80 & $-4,6 \mathrm{E}-4$ \\
\hline $20 \leq \mathrm{T} \leq 30$ & $10<U>16$ & $313,861^{* *}$ & $-0,011^{*}$ & 64,53 & 25,56 & $4,0 \mathrm{E}-4$ \\
\hline $20 \leq \mathrm{T} \leq 30$ & $16 \leq \mathrm{U} \leq 28,5$ & $319,585^{* *}$ & $-0,033^{\star *}$ & 90,19 & 29,05 & $5,0 \mathrm{E}-3$ \\
\hline $20 \leq \mathrm{T} \leq 30$ & $28,5>U<37,4$ & $308,434^{\star *}$ & $-0,029 * *$ & 94,74 & 12,37 & $-9,2 \mathrm{E}-4$ \\
\hline $30<\mathrm{T}<50$ & $10<U>16$ & $320,826 * \star$ & $-0,013^{\star \star}$ & 73,29 & 28,02 & $-3,3 \mathrm{E}-4$ \\
\hline $30<\mathrm{T}<50$ & $16 \leq \mathrm{U} \leq 28,5$ & $347,081 * *$ & $-0,038 * *$ & 92,09 & 28,87 & $6,6 \mathrm{E}-3$ \\
\hline $30<\mathrm{T}<50$ & $28,5>U<37,4$ & $285,123^{* *}$ & 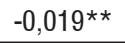 & 74,72 & 29,20 & $3,0 \mathrm{E}-5$ \\
\hline
\end{tabular}

O acréscimo de nitrato ou redução de amônio durante o processo de movimentação de água no solo foi descrito pelas equações do tipo cinética de primeira ordem (Tabela 2 e 3).

O incremento de substrato a ser contabilizado (acrescido/ retirado) em cada nó i depende das equações de regressão,

Tabela 2. Estimativa dos parâmetros $\hat{C}\left(\mathrm{mg} \mathrm{kg}^{-1}\right)$ e $\hat{\mathrm{k}}\left(\mathrm{dia}^{-1}\right)$ do modelo exponencial para simulação do amônio e as medidas estatísticas $\mathrm{R}^{2}$, RQMR e CRM

* Valores ideais: $\mathrm{RQMR}=0 ; \mathrm{CRM}=0$ 
Tabela 3. Estimativa dos parâmetros $\hat{C}\left(\mathrm{mg} \mathrm{kg}^{-1}\right)$ e $\hat{k}\left(\right.$ dia $\left.^{-1}\right)$ do modelo exponencial para simulação do nitrato e as medidas estatísticas $\mathrm{R}^{2}$, RQMR e CRM

\begin{tabular}{|c|c|c|c|c|c|c|}
\hline Temperatura $\left({ }^{\circ} \mathrm{C}\right)$ & $\begin{array}{c}\text { Teor de água } \\
\mathrm{U}\left(\mathrm{kg} \mathrm{kg}^{-1}\right)\end{array}$ & $\hat{\mathbf{C}}$ & $\hat{\mathbf{k}}$ & $\mathbf{R}^{2}$ & $\mathrm{RQMR}^{(1)}$ & $\mathrm{CRM}^{(2)}$ \\
\hline $5<T<20$ & $10<U<16$ & $17,242^{\star *}$ & $0,006^{*}$ & 99,27 & 1,09 & $-7,0 \mathrm{E}-5$ \\
\hline $5<\mathrm{T}<20$ & $16 \leq \mathrm{U} \leq 28,5$ & $16,624^{* *}$ & $0,011^{*}$ & 61,41 & 2,61 & $-5,2 \mathrm{E}-4$ \\
\hline $5<T<20$ & $28,5<U<37,4$ & $10,583^{* *}$ & $0,012^{\text {** }}$ & 80,84 & 0,36 & $-1,4 \mathrm{E}-4$ \\
\hline $20 \leq \mathrm{T} \leq 30$ & $10<U<16$ & $18,752^{\star *}$ & 0,019 ** & 78,56 & 3,13 & $-1,5 \mathrm{E}-3$ \\
\hline $20 \leq \mathrm{T} \leq 30$ & $16 \leq \mathrm{U} \leq 28,5$ & $11,519 * *$ & $0,080^{\text {** }}$ & 99,34 & 4,34 & $-1,3 \mathrm{E}-2$ \\
\hline $20 \leq \mathrm{T} \leq 30$ & $28,5<U<37,4$ & $4,614^{* *}$ & $0,034^{* *}$ & 97,52 & 0,36 & $-1,8 \mathrm{E}-3$ \\
\hline $30<\mathrm{T}<50$ & $10<U<16$ & $16,489 * *$ & $0,033^{\star *}$ & 94,27 & 0,52 & $5,3 \mathrm{E}-4$ \\
\hline $30<\mathrm{T}<50$ & $16 \leq \mathrm{U} \leq 28,5$ & $8,028^{\text {ns }}$ & $0,107^{*}$ & 87,63 & 6,53 & $-2,6 \mathrm{E}-2$ \\
\hline $30<\mathrm{T}<50$ & $28,5<U<37,4$ & $3,193 \bullet$ & 0,088 ** & 89,96 & 9,93 & $-4,4 \mathrm{E}-2$ \\
\hline
\end{tabular}

** Significativo a 1\% de probabilidade pelo teste t; * significativo a 5\% de probabilidade; • significativo a 10\% de probabilidade; ns - não significativo; R² - coeficiente de determinação; (1) RQMR - a raiz do quadrado médio do resíduo; (2) CRM - coeficiente residual de massa

válidas para o intervalo médio de temperatura e umidade para as quais foram geradas, ou seja, valores médios em relação aos tratamentos (temperatura e umidade) estabelecidos.

Para valores de temperatura do solo acima de $50{ }^{\circ} \mathrm{C}$ ou abaixo de $5^{\circ} \mathrm{C}$ ou, ainda, para teores de água acima da capacidade de campo ou abaixo do ponto de murcha, a taxa (k) dos modelos exponenciais parametrizados assume valor nulo, haja vista que a atividade metabólica dos microrganismos é baixa e desprezível (Victoria et al., 1992).

Nas Tabelas 2 e 3 tem-se os coeficientes estatísticos $R^{2}$, RQMR e CRM empregados para avaliar os modelos que consideram as transformações do nitrogênio no solo.

Nas simulações para estimativa da concentração do nitrato e amônio, considerou-se o termo sem fonte de nitrogênio (SFN) e com fonte de nitrogênio (CFN), para o $\mathrm{NO}_{3}{ }^{-}$, e sem sumidouro nitrogênio (SSN) e com sumidouro de nitrogênio (CSN), para o $\mathrm{NH}_{4}{ }^{+}$somente nos primeiros $20 \mathrm{~cm}$ de profundidade.

Os modelos exponenciais ajustados para nitrato e amônio apresentaram valores de CRM negativos (Tabela 2 e 3), induzindo à subestimação da concentração do substrato ao simularem o transporte de solutos no perfil, pelo modelo SIMASS-C.

O erro na estimativa do substrato pelo modelo (RQMR) foi maior para amônio e menor para o nitrato (Tabela 2), acarretando maiores erros nos perfis de concentração de amônio simulados, pelo SIMASS-C.

Embora o valor de $\mathrm{R}^{2}$ tenha sido elevado para modelos estimados para nitrato e amônio, os valores de RQMR foram maiores para o modelo relativo ao amônio, evidenciando que o $\mathrm{R}^{2}$ sozinho não tem muito significado (Regazzi, 2003).

Nas Figuras 2A e 2B tem-se os perfis de concentração do amônio e nitrato, respectivamente, observados experimentalmente e simulados pelo modelo SIMASS-C, 7 dias após o início do experimento para o solo arenoso.

A concentração de $\mathrm{NH}_{4}{ }^{+}$foi superestimada nos dois solos analisados, havendo ligeira melhoria ao se considerar o termo CFN e CSN; por outro lado, o $\mathrm{NO}_{3}{ }^{-}$foi subestimado no solo arenoso, enquanto para o solo argiloso houve bom ajuste, conforme RQMR e CRM (Tabela 4).

Analisando-se os perfis apresentados nas Figuras 2A, 2B, 3A e 3B, concluiu-se que a inclusão das reações biológicas do nitrogênio proporcionou pequena melhoria na estimativa da concentração de $\mathrm{NH}_{4}{ }^{+}$e $\mathrm{NO}_{3}{ }^{-}$, no entanto, os perfis simulados para nitrato indicaram melhores resultados em relação àqueles realizados para amônio; uma fonte de erro pode ser atribuída ao maior valor de RQMR obtido na estimativa da função exponencial para o amônio (Tabela 2 e 3).

Erros na estimativa da temperatura do solo pelo modelo analítico proposto, implicam em escolha errada das taxas de transformação do substrato $\left(\mathrm{NO}_{3}{ }^{-}\right.$e $\left.\mathrm{NH}_{4}{ }^{+}\right)$. Variações positivas na temperatura e umidade do solo, por exemplo, tendem a aumentar a taxa de nitrificação, gerando maior acúmulo de nitrato.

A. Concentração de amônio $\left(\mathrm{mg} \mathrm{L}^{-1}\right)$

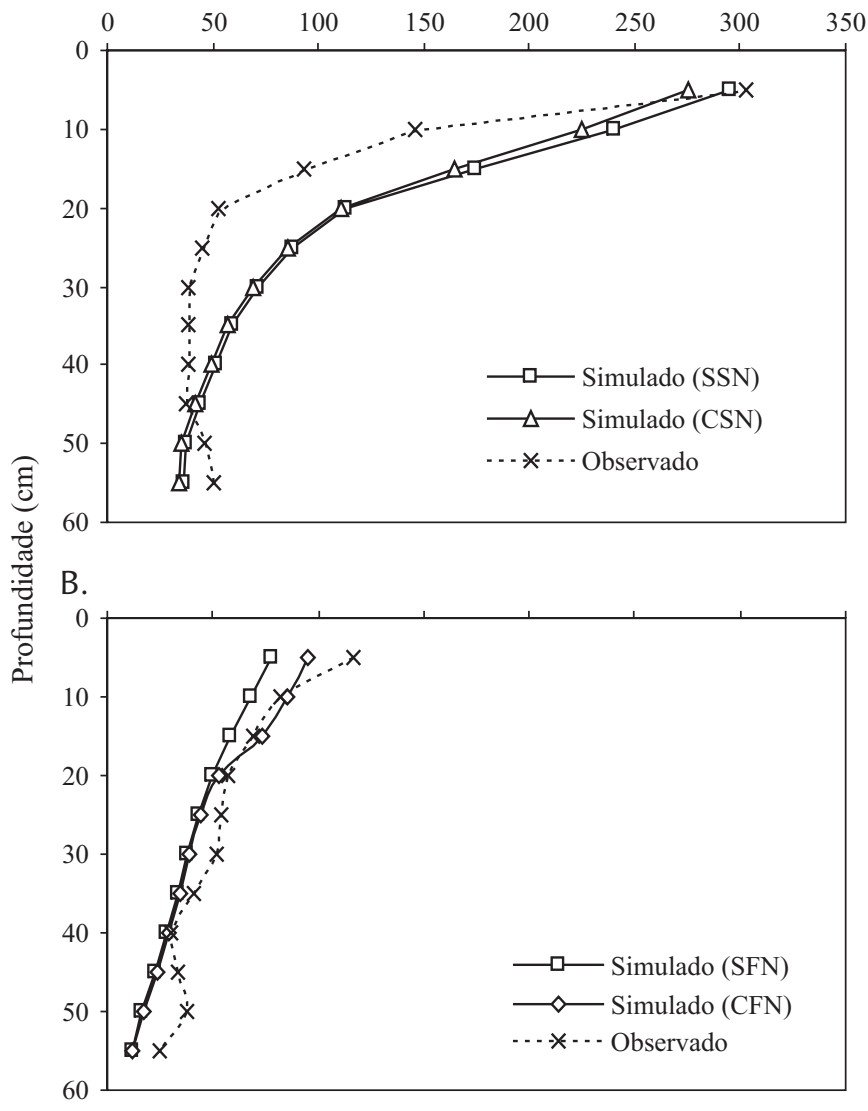

Figura 2. Perfis de concentração de $\mathrm{NH}_{4}{ }^{+}(\mathrm{A})$ e $\mathrm{NO}_{3}^{-}$(B) em solo arenoso, observados e simulados pelo modelo SIMASS-C, sem fonte de N (SFN) e com fonte de $\mathrm{N}$ (CFN), para o $\mathrm{NO}_{3}{ }^{-}$, e sem sumidouro $\mathrm{N}$ (SSN) e com sumidouro de $\mathrm{N}(\mathrm{CSN})$, para o $\mathrm{NH}_{4}{ }^{+}, 7$ dias após o início do experimento 
A.

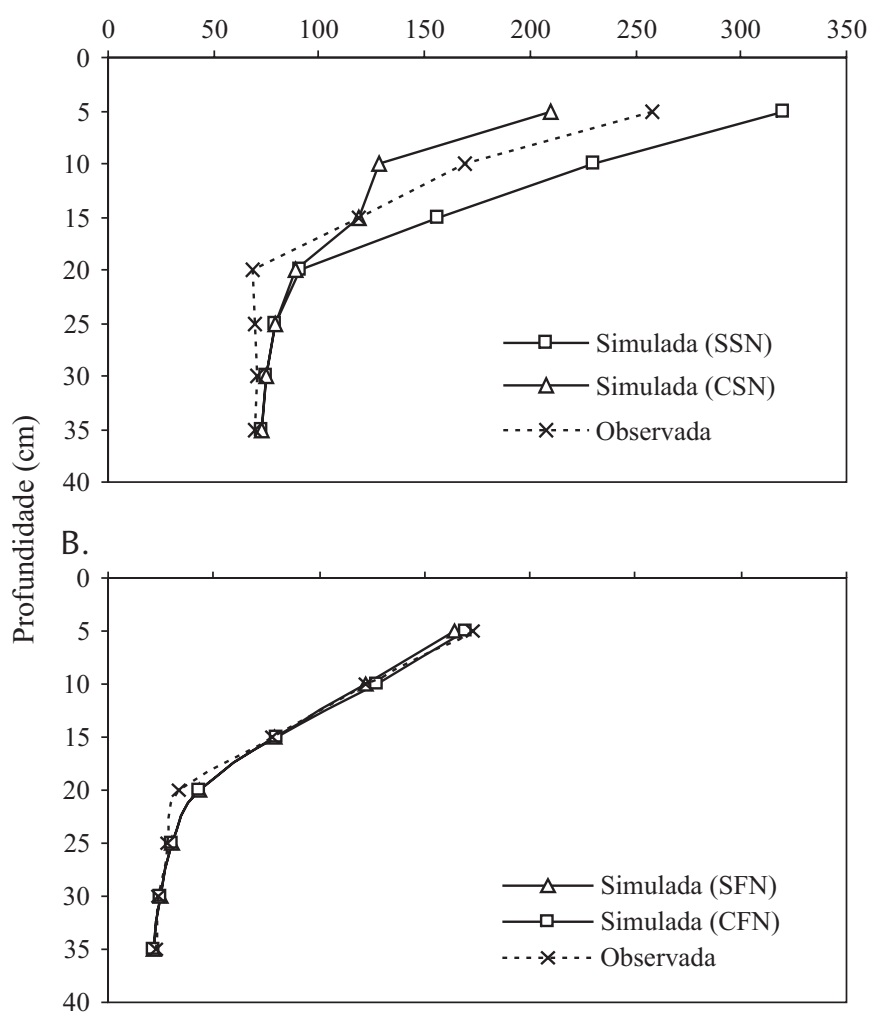

Figura 3. Perfis de concentração de $\mathrm{NH}_{4}{ }^{+}$(A) e $\mathrm{NO}_{3}^{-}$(B) em solo argiloso, observados e simulados pelo modelo SIMASS-C, sem fonte de $\mathrm{N}$ (SFN) e com fonte de $\mathrm{N}$ (CFN), para o $\mathrm{NO}_{3}{ }^{-}$, e sem sumidouro $\mathrm{N}$ (SSN) e com sumidouro de $\mathrm{N}(\mathrm{CSN})$, para o $\mathrm{NH}_{4}^{+}, 7$ dias após o início do experimento

As simulações para o $\mathrm{NH}_{4}{ }^{+}$mostraram maiores erros (Tabela 4), de acordo com os valores de RQMR, entre os dados simulados e os observados, em razão da magnitude dos dados.

Tabela 4. Valores dos coeficientes estatísticos RQMR, CRM e EF usados para avaliação do modelo SIMASS-C quanto à distribuição de nitrato e amônio no perfil do solo

\begin{tabular}{ccccc}
\hline Solo & Íon & RQMR $^{*}$ & CRM $^{*}$ & EF $^{*}$ \\
\multirow{2}{*}{ Arenoso } & nitrato & 11,26 & 0,14 & 0,91 \\
& amônio & 45,41 & $-0,31$ & 0,86 \\
\hline \multirow{2}{*}{ Argiloso } & nitrato & 4,40 & $-0,01$ & 0,99 \\
& amônio & 43,60 & 0,33 & 0,79 \\
\hline
\end{tabular}

* Valores ideais: $\mathrm{RQMR}=0 ; \mathrm{CRM}=0 \mathrm{e} \mathrm{EF}=1$

A eficiência do modelo também se mostrou satisfatória na estimativa das concentrações de $\mathrm{NO}_{3}{ }^{-}$e $\mathrm{NH}_{4}{ }^{+}$nas duas simulações, visto que os resultados de EF foram próximos de 1, exceto para a segunda simulação realizada para o nitrato, em que o valor de EF foi negativo, indicando baixa eficiência do modelo; entretanto, recomenda-se estudar outros modelos para descrever as transformações biológicas sofridas pelo nitrogênio.

Exibem-se, nas Figuras 4 e 5, os perfis de temperatura do solo gerados a partir do modelo SIMASS-C.

Observou-se uma amplitude térmica pequena e de $7,8^{\circ} \mathrm{C}$

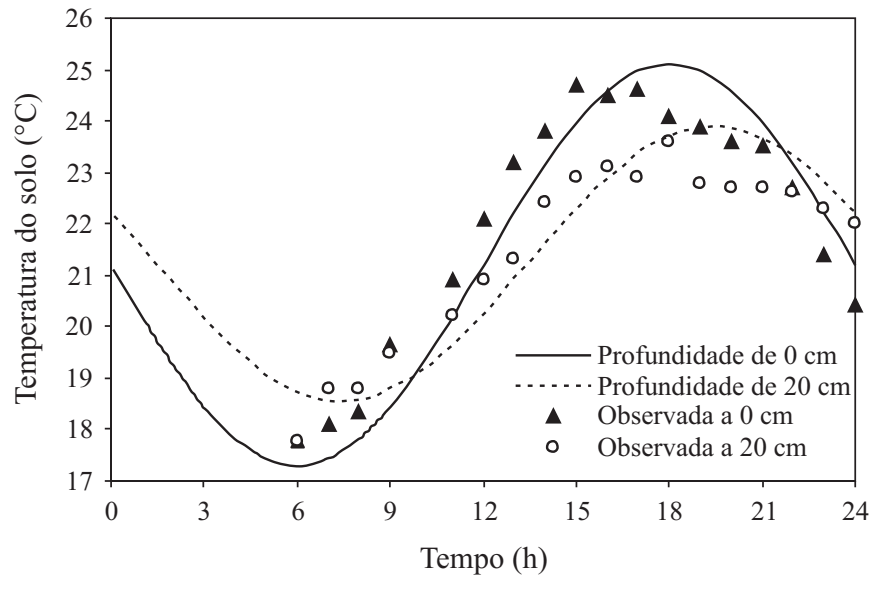

Figura 4. Perfis de temperatura do solo observados experimentalmente e simulados pelo SIMASS-C ao longo de $24 \mathrm{~h}$

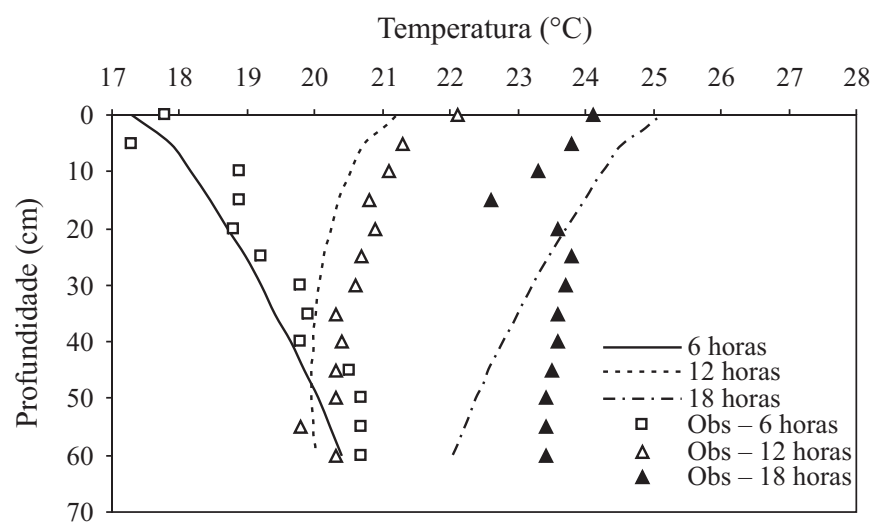

Figura 5. Perfis de temperatura do solo observados experimentalmente e simulados pelo SIMASS-C em profundidade

para a data simulada; coerente, no entanto, pois a radiação solar também foi pequena haja vista que o experimento foi conduzido em laboratório. O erro máximo na estimativa da temperatura do solo para o dia simulado foi de foi $1,38^{\circ} \mathrm{C}$ no tempo e de $1,9{ }^{\circ} \mathrm{C}$, em profundidade (Figuras 4 e 5); além disso, verificou-se que a temperatura do solo se tornou praticamente constante a partir de $35 \mathrm{~cm}$ de profundidade, e, nas Figuras 4 e 5, que o modelo analítico tendeu a subestimar a temperatura do solo, a partir das 9 h da manhã, até entre 16 e $17 \mathrm{~h}$ justamente no período de incidência de radiação solar a partir das 17 h, o modelo tendeu a superestimar a temperatura do solo.

O modelo proposto permitiu descrever a inversão de gradiente térmico; por exemplo, na Figura 5, às 6 h da manhã a temperatura observada e simulada foi menor na superfície que em profundidades do solo, coerente com o comportamento físico do fenômeno, isto é, o solo perdeu calor a partir da superfície; assim, ele esfriou durante a noite, de forma que essa perda de calor foi maior na superfície; e menor em profundidade; de maneira inversa, quando a radiação começou a incidir na superfície o aquecimento se deu de forma mais rápida na superfície e menor em profundidade.

Ao possibilitar simulações em condições de solo estratificado, o modelo tornou-se mais realístico e flexível. 


\section{CONCLUSÕES}

1. O modelo exponencial selecionado apresentou bom ajuste aos dados experimentais, tanto para a simulação do nitrato quanto para o amônio.

2. As equações cinéticas no modelo SIMASS-C, que consideram as reações do nitrogênio no solo, resultaram em pequena melhoria na estimativa da concentração de nitrato e amônio no solo, justificando o estudo de outros modelos.

3. A temperatura do solo foi estimada com boa precisão utilizando-se o modelo analítico proposto.

\section{LITERATURA CITADA}

Azevedo, A. S.; Kanwar, R. S.; Singh, P.; Pereira, L. S. Movement of $\mathrm{NO}_{3}{ }^{-}$and atrazine through soil columns as affected by lime application. Transactions of the Asae, St. Joseph, v.39, n.3, p.937-945, 1996.

Borges Júnior, J. F.; Ferreira, P. A. Equações e programa computacional para cálculo do transporte de solutos do solo. Revista Brasileira de Engenharia Agrícola e Ambiental, Campina Grande, v.10, n.3, p.604-611, 2006.

Carsel, R. F.; Mullins, J. A.; Scarbrough, J. E.; Ivery, A. M. PRZM - A model for predicting pesticide fate in the crop root unsaturated soil zones: User's manual for release 2.0. Athens. 275p.1984.

Corrêa, M. M. Desenvolvimento e teste de modelo de transporte unidimensional de solutos no solo. Viçosa: UFV, 2001.104p. Tese Doutorado

Costa, S. N. Desenvolvimento de um modelo computacional para simular o transporte de água e solutos no solo sob condições de escoamento não permanente na vertical. Viçosa: UFV, 1998. 153p. Tese Doutorado

Dourado Neto, D.; Nielsen, D. R.; Hopmans, J. W.; Reichardt, K.; Bacchi, O. O. S. Software to model soil water retention curves (swrc, version 2.00). Scientia Agrícola, Piracicaba, v.57, n.1, p.191-196, 2000.

Elias, E. A. Modelagem analítica do perfil de temperatura do solo. Piracicaba: ESALQ, 2004. 67p. Tese Doutorado

Hansen, S. H.; Jensen, E.; Nielsen, N. E.; Svenden, H. Daisy Soil plant atmosphere system model. http://www. dina.kvl.dk/ abraham/daisy. 04 Fev. 1990.

Hillel, D. Fundamentals of soil physics. Orlando: Academic Press, 1980. 362p.

Loague, K.; Green, R. E. Statistical and graphical methods for evaluating solute transport models: overview and application. Journal of Contaminant Hydrology, Amsterdam, n.7, n.1, p.51-73, 1991.
McLaren, A. D. Steady state studies of nitrification in soil: theoretical considerations. Soil Science Society American Proceedings, Madison, v.33, n.3, p.273-275, 1969.

Mengel, K. Dynamics and availability of major nutrients in soils. In: Stewart, B. A. (ed.). Advances in soil science, v.2. New York: Springer-Verlag, 1996. p.65-115.

Misra, C.; Nielsen, D. R.; Biggar, J. W. Nitrogen transformations in soil during leaching, I. theoretical considerations. Soil Science Society American Proceedings, Madison, v.38, n.3, p.289-293, 1974.

Regazzi, A. J. Teste para verificar a igualdade de parâmetros e da identidade de modelos de regressão não-linear. Revista Ceres, Viçosa, v.50, p.9-26, 2003.

Sierra, J. Temperature and soil moisture dependence of $n$ mineralization in intact soil cores. Soil Biology and Biochemistry, Oxford, v.29, n.9, p.1557-1563, 1997.

Sierra, J. Nitrogen mineralization and nitrification in a tropical soil: effects of fluctuating temperature conditions. Soil Biology and Biochemistry, Oxford, v.34, n.8, p.12191226, 2002.

Spadotto, C. A. Comportamento e destino ambiental de herbicidas. Comitê do meio ambiente, sociedade brasileira da ciência das plantas daninhas. http://www.cnpma.embrapa.br/herbicidas. 28 Mai. 2003.

Starr, J. L. Assessing nitrogen movement in the field. In: chemical mobility and reactivity in soil systems. Soil Science Society of America, Madison, n.11. cap.6, p.79-92. 1983.

Strong, D. T.; Fillery, I. R. P. Denitrification response to nitrate concentrations in sandy soils. Soil Biology and Biochemistry, v.24, p.945-954, 2002.

van Genucthen, M. A closed-form equation for predicting the hydraulic conductivity of unsaturated soils. Soil Science Society American Journal, Madison, v.44, n.4, p.892-898, 1980.

Victoria, R. L.; Piccolo, M. C.; Vargas, A. A. T. O ciclo do nitrogênio, in: Cardoso, E. J. B. N.; Tsai, S. M.; Neves, M. C. P. Microbiologia do solo. Campinas: Sociedade Brasileira de Ciência do Solo, cap. 9, p.105-119, 1992.

Wagenet, R. J.; Rao, P. S. C. Modeling pesticide fate in soils, in: Cheng, H. H. Pesticides in the soil environment: processes, impacts and modeling: Soil Science Society of America, Madison, v.14, n.3, p.351-359, 1990.

Wierenga, P. J.; Wit, C. T. Simulation of heat transfer in soils. Soil Science Society American Proceedings, Madison, v.34, n.3, p.845-848, 1970.

Wu, J.; Nofziger, D. L. Incorporating temperature effects on pesticide degradation into a management model. Journal Environment Quality, Madison, v.28, n.2, p.92-100, 1999. 